ORIGINAL ARTICLE

\title{
Acute Kidney Injury is A Risk Factor among Type 2 Diabetic Patients after UTI due to Extended-Spectrum Beta-Lactamase Producing Organisms
}

\author{
KASHIF ALI SAMIN 1 , SARA MALIK², SIDRA SADIQ ${ }^{3}$, TALHA RASHEEQ ${ }^{4}$, NISAR KHAN SAJID ${ }^{5}$, WAHEED IQBAL ${ }^{6}$ \\ ${ }^{1}$ Assistant Professor Family Medicine, Khyber Medical University, Peshawar \\ ${ }^{2}$ Assistant Consultant Medicine, Mukhtar A Sheikh Hospital, Multan \\ ${ }^{3}$ WMO Internal Medicine Govt. Shahbaz Sharif Hospital, DHQ Multan \\ ${ }^{4}$ Associate Professor Medicine, Bakhtawar Amin Medical College, Multan \\ ${ }^{5}$ Associate Professor of Pediatric Medicine, Aziz Fatima Medical and Dental College, Faisalabad \\ ${ }^{6}$ Associate Professor Department of Medicine, Divisional Headquarters Teaching Hospital Mirpur/ Mohtarma Benazir Bhutto Shaheed \\ Medical College, Mirpur \\ Corresponding author: Dr. Kashif Ali Samin, E-mail: kashif@kmu.edu.pk, Phone:+923009590555
}

\begin{abstract}
Background: Urinary tract infections (UTIs) are very communal, and patients with diabetics develop UTIs more frequently. Acute kidney injury (AKI) can be a complication of UTI. This study is designed to determine whether Urinary tract infections because of extended-spectrum beta-lactamase (ESBL) could be a risk factor of acute kidney injury in individuals with type-II diabetes mellitus.

Methods: This case study was conducted in the Outpatient department of Diabetes Hospital Peshawar and Nishter Hospital Multan for duration of six months from August 2020 to January 2021. People of type II diabetes were assessed with culture confirmed UTI. The cases of UTI patients complicated with AKI were included in the study group, and people without AKI were taken as a control group. ESBLs positivity from isolated organisms have been assessed as risk factors for AKI. A total of 140 subjects were selected with equal distribution in two groups. The group A has UTI complicated with AKI and group B included has patients without AKI but with UTI.

Results: UTI was diagnosed in 140 cases among type-Il diabetic patients with 2:4 male to female ratio. The duration and mean age of diabetes mellitus were $8.60 \pm 5.35 a n d 55.80 \pm 14.10$ years, correspondingly. The strongest common etiological factor was Escherichia coli $(60.7 \%)$, trailed by Klebsiella pneumoniae (11.4\%). In ESBL positive organism; E. coli was present in $81.4 \%$ and $\mathrm{K}$. pneumoniae in $10 \%$ of individuals. Of the $140 \mathrm{UTI}$ cases, AKI was observed in $70(50 \%)$; out of which $48(62.5 \%)$ were ESBL-positive microorganisms and 22 $(22 / 70,31.43 \%)$ for non-ESBL microorganisms.

Conclusions: Nearly50\% of the patients with type-Il diabetes mellitus and UTI had ESBL-positive microorganisms as etiological mediators in this analysis. Owing to the presence of ESBL-positive microorganisms, UTI was the main cause of AKI and is a strong risk factor.

Keywords: ESBL, Acute kidney injury, UTI, type 2 diabetes, risk factor.
\end{abstract}

\section{INTRODUCTION}

In adults; the very communal bacterial infection is Urinary tract infection. Women and individuals with diabetes have a higher risk of Urinary tract infection ${ }^{1-2}$. Klebsiella pneumoniae and Escherichia coli are the 2 of the most communal sources of UTIs in the world ${ }^{3-4}$. Strains, which produce broad-spectrum beta-lactamase (ESBL), is the growing issue, and patients with diabetes in long-term and poor glycemic control are at augmented jeopardy of UTIs because of ESBL-positive organisms ${ }^{5-6}$. Urinary tract infection can be complicated by sepsis and acute kidney injury ${ }^{7}$. This analysis was planned to determine whether Urinary tract infection occurred because of ESBL-positive microorganisms must be measured a risk feature for acute kidney injury in patients with type-Il diabetes.

\section{METHODS}

This case study was registered in Pakistan and conducted in Outpatient department of Diabetes Hospital Peshawar and Nishter Hospital Multan for duration of six months from August 2020 to January 2021.. The patients admitted with clinical analysis of UTI were included for the study. A clean urine collection procedure was used (morning samples preferably). In the microbiology laboratory of the Hospital; the collected samples were sent within 30 minutes and then inoculated with blood agar and Mac Conkey agar within two hours. Significant positive cultures were included in the study (large number of colonies> 1x105 Units of colonies/ ml). Pregnant women, Catheterized patients and patients with insufficient or no growth and with candida culture in the urine culture were not included. The organism's growth was further assessed by the characteristics of their colonies and biochemical tests, with motility indole urea, (MIU) Motole Indole urea (MIU), Simon's citrate and triple sugar iron (TSI) test. The included samples were transferred for antibiotic susceptibility determination on Muellar-Hilton ( $\mathrm{MH})$ agar using the KibryBauer disc susceptibility test. The Double Disc Synergy Test was used to assess the ESBL-positivity phenotypically. In addition to the urine culture, the subjects were assessed clinically and additional necessary laboratory investigations were performed. It was determined whether patients developed AKI. Improving Global Outcomes (KDIGO) Clinical Practice Guide 2012 guidelines were used for the AKI Diagnosis of Renal Disease. The cases with UTI complicated by acute kidney injury were subsequently treated and included in the experimental group and UTI without AKI were included as control. Lastly, patients with ESBL-positive organisms were 
assessed as risk factors for acute kidney injury in study participants. SPSS 20.0 was used for data analysis; the value $<0.05 P$ was considered significant. The outcomes are accessible in the tables.

\section{RESULTS}

UTI was diagnosed in 140 cases among type-II diabetic patients with 2:4 male to female ratio. The duration and mean age of diabetes mellitus were $8.60 \pm 5.35$ and55.80 \pm 14.10 years, correspondingly.
The patient demographic features are given in Table-1

\begin{tabular}{|l|l|l|}
\hline Demographic Features & ESBL Group & Non-ESBL Group \\
\hline Males (85) & 55 & 30 \\
\hline Females (55) & 35 & 20 \\
\hline Total & 90 & 50 \\
\hline Education Level & 34 & 48 \\
\hline Primary & 23 & 15 \\
\hline Secondary & 13 & 7 \\
\hline Graduate
\end{tabular}

The studied subjects Base-line characteristics are given in Table-2

\begin{tabular}{|l|l|l|l|l|}
\hline Features & Total $(\mathrm{N}=140)$ & UTI cases with AKI $(\mathrm{n}=70)$ & $\begin{array}{l}\text { UTI cases without AKI) } \\
(\mathrm{n}=70) \text { Control group }\end{array}$ & $p$ value \\
\hline Age in Years (Mean) & $55.80 \pm 14.10$ & $53.20 \pm 12.21$ & $55.30 \pm 11.60$ & 0.69 \\
\hline M: F ratio & $1: 2.4$ & $35: 45$ & $30: 40$ & 0.23 \\
\hline DM (Mean duration in years) & $8.60 \pm 5.35$ & $9.20 \pm 5.31$ & $8.25 \pm 5.42$ & 0.36 \\
\hline Mean HbA1c (\%) & $8.50 \pm 1.80$ & $9.1 \pm 1.80$ & $8.70 \pm 2.09$ & 0.690 \\
\hline
\end{tabular}

The strongest common etiological factor was Escherichia coli $(60.7 \%)$, trailed by Klebsiella pneumoniae $(11.4 \%)$. In ESBL positive organism; E. coli was present in $81.4 \%$ and $\mathrm{K}$. pneumoniae in $10 \%$ of individuals. Of the 140 UTI cases, AKI was observed in $70(50 \%)$; out of which 48 $(62.5 \%)$ were ESBL-positive microorganisms and 22 $(22 / 70,31.43 \%)$ for non-ESBL microorganisms.

Etiological mediatorsamid the study subjects $(N=140)$ given in Table-3

\begin{tabular}{|l|l|l|l|}
\hline Bacteria & ESBL-positive & Non-ESBL & \multicolumn{1}{|c|}{ Total } \\
\hline K. pneumoniae & $7(5)$ & $9(6.1)$ & $16(11.4)$ \\
\hline E. coli & $57(40.7)$ & $28(20.6)$ & $85(60.7)$ \\
\hline Citrobacter & - & $2(1.42)$ & $2(1.42)$ \\
\hline $\begin{array}{l}\text { Pseudomonas } \\
\text { spp. }\end{array}$ & - & $2(1.42)$ & $2(1.42)$ \\
\hline MRSA Staph & - & $5(3.6)$ & $5(3.6)$ \\
\hline Staphylococcus & - & $2(1.42)$ & $2(1.42)$ \\
\hline Acinetobacter & - & $11(7.85)$ & $11(7.85)$ \\
\hline Enterococcus & - & $9(6.4)$ & $9(6.4)$ \\
\hline Streptococcus & - & - & $2(1.42)$ \\
\hline Citrobacter & $3(2.14)$ & $2(1.42)$ & $5(3.6)$ \\
\hline Enterobacter & $3(2.14)$ & --- & $3(2.14)$ \\
\hline Total & 70 & 70 & $140(100)$ \\
\hline
\end{tabular}

There were no significant differences between the significant control group in terms of age $(p=0.69)$, sex $(p=$ $0.23)$, duration of diabetes $(p=0.36)$, and glucose hemoglobin $(\mathrm{HbA1c})(p=0.69)$. The ESBL positivity seems to be a substantial risk factor for acute kidney injury between studies(Table-4).

Estimation of UTI with ESBL-positive organisms as the AKI riskfactors amid the study subjects $(\mathrm{N}=140)$ given in Table-4

\begin{tabular}{|l|l|l|l|}
\hline Bacteria & $\begin{array}{l}\text { ESBL- } \\
\text { positive }\end{array}$ & Non- ESBL & Total \\
\hline ESBL-positive (70) & 48 & 22 & 70 \\
\hline Non-ESBL (70) & 20 & 50 & 70 \\
\hline & 68 & 72 & 140 \\
\hline
\end{tabular}

\section{DISCUSSION}

The description of acute kidney injury has been developed in several steps, and KDIGO has distinct AKI that meets one or more of the subsequent three criteria's: 48-hour rise in serum creatinine over $0.3 \mathrm{mg} / \mathrm{dL}$, serum creatinine 1.5 times higher of the baseline in 7 days, the urine output decreased to $0.5 \mathrm{ml} / \mathrm{kg} / \mathrm{h}$ in less than six hours ${ }^{7-8}$. This is the newest and maximum applied $\mathrm{AKI}$ criterion and we appliedthis criterion to define AKI in this research. Our primary focus is on the 1st two measures grounded on serum levels of creatinine ${ }^{9-10}$. Acute kidney injury is communal in hospitals and most often in intensive care units (ICUs). Diabetic patients are correspondingly at higher jeopardy of acute kidney injury. Previous CKD and AKI positive history are risk factors for acute kidney injury ${ }^{11}$. Urinary tract infection in AKI can make things difficult ${ }^{12-13}$. Age, diabetes, subterranean kidney disease, upper UTI are risk factors for the UTI. In our studies, we assessed the positive ESBL of microbes influencing UTI as AKI risk factor $^{14-15}$. Due to the presence of ESBL-positive microorganisms in Pakistan, the number of UTI is increasing, and Rahim MA et al. Report that women's who are diabetic for a long-time and poor glycemic control and ESBL-positive organisms are the risk factors for UTIs. A comparable result was made in additional studies. In another study, continued hospitalization and multiple UTIs and antibiotic are factors for ESBL-positive microorganisms causing UTI ${ }^{16-17}$. UTIs because of ESBL-positive organisms may also affect the function of recipients of kidneys and kidney transplants. We found that a positive ESBL test in type 2 diabetic patients was a substantial AKI risk factor. This analysis had some probable limits. The studied time was 6 months, single center study and the small sample size. We have not assessed additional risk factors for AKI in baseline renal function, such as treatment with angiotensin blockers such as ARBs, ACEl or the hydration standing of patients taking medications using nephrotoxic antibiotics ${ }^{18-19}$. Taking all these mixtures into account, a wider, multi-center analysis will provide much dependable conclusions to the investigative questions raised in this analysis $^{21-22}$. UTI in diabetic patients with complicated AKI deserve follow-up for an extended period of time kidney 
disease. Taking this fact into account also served us in this particular investigation.

\section{CONCLUSION}

Finally, UTI can be said to increase due to the presence of ESBL-positive microorganisms; almost half of the patients with type-Il diabetes have the UTI in this analysis. Because of ESBL-positive uropathogens, the UTI appears to be a major risk factor in type-II diabetics causing AKI. Virtuous glycemic control and over-all preventive measures in the UTI may be supportive to prevent UTI caused by ESBLpositive organisms and thus decrease the chances of $\mathrm{AKI}$ in type-II diabetic patients.

\section{REFERENCES}

1. Kim JH, Lee J, Kim DH, Park JY, Lee H, Kang HG, Ahn YH. Maternal antibiotic exposure during pregnancy is a risk factor for community-acquired urinary tract infection caused by extended-spectrum beta-lactamase-producing bacteria in infants. Pediatric Nephrology. 2021 Jun 25:1-8.

2. Ahn ST, Da Eun Han DH, Kim JW, Park HS, Du Geon Moon MM. Single-dose amikacin plus 7 days of amoxicillin/clavulanate to treat acute cystitis caused by extended-spectrum beta-lactamase-producing Escherichia coli: A retrospective cohort study. Investigative and Clinical Urology. 2021 May;62(3):310.

3. Robles-Torres JI, Ocaña-Munguía MA, Arrambide-Herrera JG, Martínez-Fernández AM, Romero-Mata R, GómezGuerra LS. What is the prognosis of emphysematous pyelonephritis associated with extended-spectrum betalactamases producing microorganisms?. Asian Journal of Urology. 2021 May 5.

4. Kotalwar RR, Karande GM, Purandare VB, Deshmukh HS, Pakale AM, Unnikrishnan AG. Recurrent and complicated urinary tract infection in type 2 diabetes: Case series. Journal of Diabetology. 2021 Apr 1;12(2):228.

5. Chen WC, Hung CH, Chen YS, Cheng JS, Lee SS, Tseng FC, Cheng MF, Wang JL. Bloodstream Infections Caused by Extended-Spectrum Beta-Lactamase-Producing Escherichia coli in Patients with Liver Cirrhosis. Pathogens. 2021 Jan;10(1):37.

6. Quan J, Dai H, Liao W, Zhao D, Shi Q, Zhang L, Shi K, Akova M, Yu Y. Etiology and prevalence of ESBLs in adult community-onset urinary tract infections in East China: a prospective multicenter study. Journal of Infection. 2021 Jun 9.

7. NazliZeka A, Avkan-Oguz V, Irmak C, ErenKutsoylu O, Alp Cavus S, Kuruüzüm Z, Ergon MC. Daily inpatient ertapenem therapy can be an alternative to hospitalization for the treatment of complicated urinary tract infections during the COVID-19 pandemic. International journal of clinical practice. 2021 Apr 20:e14230.

8. Kizilates F, Yakupogullari Y, Berk H, Oztoprak N, Otlu B. Risk factors for fecal carriage of extended-spectrum betalactamase-producing and carbapenem-resistant Escherichia coli and Klebsiella pneumoniae strains among patients at hospital admission. American Journal of Infection Control. 2021 Mar 1;49(3):333-9.

9. Sule IO. The Causes and Control Measures of Extended Spectrum Beta-Lactamase Producing Enterobacteriaceae in Long-Term Care Facilities (Doctoral dissertation, Walden University).

10. Garcia-Bustos V, Escrig Al, López CC, Estellés RA, Jerusalem K, Cabañero-Navalón MD, Massó VM, SigonaGiangreco IA, Sahuquillo-Arce JM, Hernández IC, Lletí MS. Prospective cohort study on hospitalised patients with suspected urinary tract infection and risk factors por multidrug resistance. Scientific reports. 2021 Jun 7;11(1):10.

11. Katip W, Yoodee J, Uitrakul S, Oberdorfer P. Efficacy of loading dose colistin versus carbapenems for treatment of extended spectrum beta lactamase producing Enterobacteriaceae. Scientific Reports. 2021 Jan 8;11(1):18.

12. Hyun M, Lee JY, Kim HA. Differences of Virulence Factors, And Antimicrobial Susceptibility According To Phylogenetic Group In Uropathogenic Escherichia Coli Strains Isolated From Korean Patients.

13. Ibrahem $\mathrm{KH}$, Ali FA, Sadiq AH, Abdulkarim AS, Rasul DT, Hussein ET. FREQUENCY OF EXTEND SPECTRUM BETA LACTAMASE IN MULTIRESISTANCE ESCHERICHIA COLI AND KLEBSIELLA PNEUMONIA ISOLATED FROM KIDNEY TRANSPLANTATION PATIENTS. Plant Archives. $2021 ; 21(1): 1042-51$.

14. Tavakoli-Far B, Pourhatami F, Gharavi MJ. Prevalence and antimicrobial resistance properties of 3 Extended-spectrum beta-lactamases (ESBL) producing 4 Escherichia coli isolated from the cases of Urinary Tract 5 Infections 6.

15. Bedenić $B$, Meštrović T. Mechanisms of Resistance in GramNegative Urinary Pathogens: From Country-Specific Molecular Insights to Global Clinical Relevance. Diagnostics. 2021 May;11(5):800.

16. Kasew D, Eshetie S, Diress A, Tegegne Z, Moges F. Multiple drug resistance bacterial isolates and associated factors among urinary stone patients at the University of Gondar Comprehensive Specialized Hospital, Northwest Ethiopia. BMC urology. 2021 Dec;21(1):1-1.

17. Zbinden R, Hasse B. Risks Factors for Infections with Extended-Spectrum Beta-Lactamase-Producing Escherichia coli and Klebsiella pne...

18. Rozenkiewicz D, Esteve-Palau E, Arenas-Miras M, Grau S, Duran X, Sorlí L, Montero MM, Horcajada JP. Clinical and Economic Impact of Community-Onset Urinary Tract Infections Caused by ESBL-Producing Klebsiella pneumoniae Requiring Hospitalization in Spain: An Observational Cohort Study. Antibiotics. 2021 May;10(5):585.

19. da Silva P, Lustri BC, Castilho IG, Ferreira AM, Hernandes RT, Schembri MA, Moreira CG. Genome profiling of fluoroquinolone-resistant uropathogenic Escherichia coli isolates from Brazil. Brazilian Journal of Microbiology. 2021 Jun 8:1-9.

20. Anesi JA, Lautenbach E, Tamma PD, Thom KA, Blumberg EA, Alby K, Bilker WB, Werzen A, Tolomeo P, Omorogbe J, Pineles L. Risk factors for extended-spectrum $\beta$-lactamaseproducing enterobacterales bloodstream infection among solid-organ transplant recipients. Clinical Infectious Diseases. 2021 Mar 15;72(6):953-60.

21. Martínez-Casanova J, Gómez-Zorrilla S, Prim N, Dal Molin A, Echeverría-Esnal D, Gracia-Arnillas MP, Sendra E, Güerri-Fernández R, Durán-Jordà X, Padilla E, Horcajada JP. Risk Factors for Amoxicillin-Clavulanate Resistance in Community-Onset Urinary Tract Infections Caused by Escherichia coli or Klebsiella pneumoniae: The Role of Prior Exposure to Fluoroquinolones. Antibiotics. 2021 May;10(5):582.

22. Martínez-Casanova J, Gómez-Zorrilla S, Prim N, Dal Molin A, Echeverría-Esnal D, Gracia-Arnillas MP, Sendra E, Güerri-Fernández R, Durán-Jordà X, Padilla E, Horcajada JP. Risk Factors for Amoxicillin-Clavulanate Resistance in Community-Onset Urinary Tract Infections Caused by Escherichia coli or Klebsiella pneumoniae: The Role of Prior Exposure to Fluoroquinolones. Antibiotics. 2021 May;10(5):582. 